\title{
Goldstone boson currents in a kaon condensed CFL phase
}

\author{
A. Gerhold and T. Schäfer \\ Department of Physics, North Carolina State University, Raleigh, NC 27695
}

\author{
A. Kryjevski \\ Nuclear Theory Center, Indiana University, Bloomington, IN 47408
}

\begin{abstract}
We study the stability of the kaon condensed color-flavor locked (CFL) phase of dense quark matter with regard to the formation of a non-zero Goldstone boson current. In the kaon condensed phase there is an electrically charged fermion which becomes gapless near $\mu_{s}^{(1)} \simeq 1.35 \Delta$ and a neutral fermion which becomes gapless near $\mu_{s}^{(2)} \simeq 1.61 \Delta$. Here, $\mu_{s}=m_{s}^{2} /\left(2 p_{F}\right)$ is the shift in the Fermi energy due to the strange quark mass $m_{s}$ and $\Delta$ is the gap in the chiral limit. The transition to the gapless phase is continuous at $\mu_{s}^{(1)}$ and first order at $\mu_{s}^{(2)}$. We find that the magnetic screening masses are real in the regime $\mu_{s}<\mu_{s}^{(2)}$, but some screening masses are imaginary for $\mu_{s}>\mu_{s}^{(2)}$. We show that there is a very weak current instability for $\mu_{s}>\mu_{s}^{(1)}$ and a more robust instability in a small window near $\mu_{s}^{(2)}$. We show that in the Goldstone boson current phase all components of the magnetic screening mass are real. There is a range of values of $\mu_{s}$ below $2 \Delta$ in which the magnetic gluon screening masses are imaginary but the phase is stable with respect to electrically neutral fluctuations of the gauge field.
\end{abstract}




\section{INTRODUCTION}

Understanding the response of a paired fermion system to an external pair breaking field has been an area of intense theoretical and experimental research over the last couple of years. In dense quark matter the problem arises in connection with the effect of a nonzero strange quark mass, see [1] for recent reviews. If quarks are massless and the flavor symmetry is exact then the ground state of three flavor quark matter at high baryon density is the color-flavor-locked (CFL) phase [2, 3, 4]. The CFL state involves pairing between up and down, up and strange, as well as down and strange quarks. In the real world the up and down quarks are approximately massless but the mass of the strange quark is comparable to the QCD scale parameter. At high baryon density the effect of the strange quark mass is governed by the shift $\mu_{q}=m_{q}^{2} /(2 \mu)$ of the Fermi energy due to the mass.

A non-zero $\mu_{s}$ implies a non-zero pair breaking stress on $u d$ and $d s$ pairs. Once CFL pairing is disrupted, an additional stress arises due to the different electric charges of the $u$ and $d$ quark. There are two scales that govern the response to $\mu_{s}$, the mass $m_{K}$ of the lightest strange Goldstone boson, the kaon, and the gap $\Delta$ for fermionic excitations. When $\mu_{s}$ is equal to $m_{K}$ the CFL phase undergoes a transition to a phase in which the CFL order parameter rotates in flavor space and a kaon condensate appears [5]. If $\mu_{s}>\Delta$ gapless fermion modes appear in the spectrum and the CFL pairing pattern starts to get disrupted [6, 7]. We will refer to the phase with kaon condensation as the CFLK phase, and the gapless phases as the gCFL and gCFLK phase.

Gapless fermion modes in weakly coupled pair condensates cause instabilities in currentcurrent correlation functions [8, 9] and these instabilities have been found in the gapless CFL phase [10, 11, 12]. We have suggested that the instability is resolved by the formation of a non-zero Goldstone boson current [13, 14, 15]. In our previous work we analyzed the Goldstone boson current in the kaon condensed CFL phase, but we restricted the pairing ansatz to a flavor rotation of the CFL state and did not properly implement charge neutrality in the Goldstone boson current state [13, 14]. In [15] we allowed the CFL pairing pattern to be disrupted and constructed a charge neutral solution, but we did not allow kaon condensation.

In the present work we study Goldstone boson currents in a kaon condensed CFL phase. We use a very general ansatz for the current, allow the CFL pairing pattern to be disrupted, 
and implement electric and color charge neutrality. We also compute the magnetic screening masses. Our work remains restricted to states in which the Goldstone boson current is small compared to the gap. This is expected to be the case as long as $\mu_{s}$ is close to the onset for gapless fermion modes in the spectrum. The opposite limit of a current which is large compared to the gap in analyzed in studies of the Larkin-Ovchinnikov-Fulde-Ferrell (LOFF) state in three flavor QCD [16, 17, 18, 19, 20].

\section{EFFECTIVE LAGRANGIAN AND DISPERSION LAWS}

Our starting point is an effective lagrangian for gapped fermions interacting with gauge fields $[21,22]$

$$
\begin{aligned}
\mathcal{L}= & \operatorname{Tr}\left(\chi_{L}^{\dagger}\left(i v \cdot \partial-\hat{\mu}^{L}-A_{e} Q\right) \chi_{L}\right)+\operatorname{Tr}\left(\chi_{R}^{\dagger}\left(i v \cdot \partial-\hat{\mu}^{R}-A_{e} Q\right) \chi_{R}\right) \\
& -i \operatorname{Tr}\left(\chi_{L}^{\dagger} \chi_{L} X v \cdot\left(\partial-i A^{T}\right) X^{\dagger}\right)-i \operatorname{Tr}\left(\chi_{R}^{\dagger} \chi_{R} Y v \cdot\left(\partial-i A^{T}\right) Y^{\dagger}\right) \\
& -\frac{1}{2} \sum_{a, b, i, j, k} \Delta_{k}\left(\chi_{L}^{a i} \chi_{L}^{b j} \epsilon_{k a b} \epsilon_{k i j}-\chi_{R}^{a i} \chi_{R}^{b j} \epsilon_{k a b} \epsilon_{k i j}+\text { h.c. }\right) .
\end{aligned}
$$

The notation agrees with our earlier work [15]. $\chi_{L, R}^{a i}$ are left/right handed fermions with color index $a$ and flavor index $i, A_{\mu}$ are $S U(3)_{C}$ color gauge fields, and $\hat{\mu}^{L}=M^{\dagger} /(2 \mu)$, $\hat{\mu}^{R}=M^{\dagger} M /(2 \mu)$ are effective chemical potentials induced by the quark mass matrix $M$. The trace part of $\hat{\mu}^{L, R}$ corresponds to a shift in the baryon chemical potential and will be neglected. The matrix $Q=\operatorname{diag}\left(\frac{2}{3},-\frac{1}{3},-\frac{1}{3}\right)$ is the quark charge matrix and $A_{e}$ is an electrostatic potential. The fields $X, Y$ determine the flavor orientation of the left and right handed gap terms and transform as $X \rightarrow L X C^{T}, Y \rightarrow R Y C^{T}$ under $(L, R) \in S U(3)_{L} \times S U(3)_{R}$ and $C \in S U(3)_{C}$, and $\Delta_{k}(k=1,2,3)$ are the CFL gap parameters. From the lagrangian given in equ. (11) we can read off the left handed Nambu-Gor'kov propagator,

$$
\left(\begin{array}{cc}
G_{(L)}^{+} & \Xi_{(L)}^{-} \\
\Xi_{(L)}^{+} & G_{(L)}^{-}
\end{array}\right)=\left(\begin{array}{cc}
\left(p_{0}-p\right) \mathbf{1}-\mathcal{X}_{(L), v} & \underline{\Delta} \\
\underline{\Delta} & \left(p_{0}+p\right) \mathbf{1}+\mathcal{X}_{(L),-v}^{T}
\end{array}\right)^{-1}
$$

where $p=\vec{v} \cdot \vec{p}-\mu$, with the Fermi velocity $\vec{v}$. The right handed propagator is obtained by replacing $(L)$ with $(R)$. The components of the propagator are matrices in color-flavor

space. We use a basis spanned by the Gell-Mann matrices $\lambda^{A}(A=1, \ldots, 8)$ and $\lambda_{0}=\sqrt{\frac{2}{3}} \mathbf{1}$. In this basis

$$
\underline{\Delta}^{A B}=-\frac{1}{2} \Delta_{a b} \varepsilon_{i j a} \varepsilon_{r s b} \lambda_{i r}^{A} \lambda_{j s}^{B}, \quad \Delta_{a b}=\operatorname{diag}\left(\Delta_{1}, \Delta_{2}, \Delta_{3}\right)_{a b}
$$


The right and left handed gauge field vertices are (for $Y=X^{\dagger}$ )

$$
\begin{aligned}
& \mathcal{X}_{(L), v, A B}=\frac{1}{2} \operatorname{Tr}\left[\lambda_{A}\left(\hat{\mu}^{L}+A_{e} Q\right) \lambda_{B}+i \lambda_{A} \lambda_{B} X v \cdot\left(\partial-i A^{T}\right) X^{\dagger}\right] \\
& \mathcal{X}_{(R), v, A B}=\frac{1}{2} \operatorname{Tr}\left[\lambda_{A}\left(\hat{\mu}^{L}+A_{e} Q\right) \lambda_{B}+i \lambda_{A} \lambda_{B} X^{\dagger} v \cdot\left(\partial-i A^{T}\right) X\right] .
\end{aligned}
$$

Instead of using $\lambda_{3}$ and $\lambda_{8}$ it will turn out to be convenient to use the following linear combinations,

$$
\lambda_{I}=\lambda_{3}+\frac{1}{\sqrt{3}} \lambda_{8}, \quad \lambda_{I I}=\lambda_{3}-\sqrt{3} \lambda_{8}
$$

We remark that $\left[\lambda_{I}, \lambda_{7}\right]=0$. We assume a maximal kaon condensate [5],

$$
X=\xi_{K^{0}} \equiv \exp \left(\frac{i \pi}{4} \lambda_{6}\right)
$$

and make the following ansätze for $A^{0 T}$ and $\vec{A}^{T}[23,24]$,

$$
\begin{aligned}
& A^{0 T}=-\frac{1}{2}\left[X^{\dagger}\left(\hat{\mu}^{L}+A_{e} Q\right) X+X\left(\hat{\mu}^{L}+A_{e} Q\right) X^{\dagger}\right]+\tilde{A}_{I} \lambda_{I}+\tilde{A}_{7} \lambda_{7}, \\
& \vec{A}^{T}=\frac{\vec{\jmath}}{2}\left(c_{I} \lambda_{I}-\frac{c_{0}}{\sqrt{6}} \lambda_{0}+c_{7} \lambda_{7}\right) .
\end{aligned}
$$

This particular flavor structure leads to diagonal matrices for $X A_{\mu}^{T} X^{\dagger}$ and $X^{\dagger} A_{\mu}^{T} X$. For $\tilde{A}_{7}=0$ the ansatz (9) for $\vec{A}^{T}$ is equivalent to setting

$$
X=V U \xi_{K^{0}} U^{\dagger}
$$

with

$$
U=\exp \left[-\frac{i}{4} \vec{\jmath} \cdot \vec{x} c_{7} \lambda_{I I}\right], \quad V=\exp \left[-\frac{i}{2} \vec{\jmath} \cdot \vec{x}\left(c_{I} \lambda_{I}-\frac{c_{0}}{\sqrt{6}} \lambda_{0}\right)\right],
$$

and taking the solution for $\vec{A}^{T}$ given by $\vec{A}^{T}=\frac{i}{2}\left(X^{\dagger} \vec{\partial} X+X \vec{\partial} X^{\dagger}\right)$. For $\tilde{A}_{7} \neq 0$ the computation of dispersion relations using the ansatz (10) is not straightforward because the $x$-dependence does not drop out. Therefore we will use the ansatz given by equs. (7) and (9) as our starting point. In the end we will see that the condition $\tilde{A}_{7}=0$ is indeed fulfilled, which means that the ansatz for the gauge field (9) is equivalent to the ansatz for the Goldstone boson current (10). We remark that the ansatz of Ref. [14] corresponds to $c_{0}=c_{I}=0$, $c_{7} \neq 0$, while the ansatz of Ref. [13] corresponds to $c_{0}, c_{7} \neq 0, c_{I}=0$.

We find that the tadpole diagrams with an external $A_{1,2,4,5,6, I I}$ vanish identically for any values of the parameters of our ansatz. Therefore the corresponding neutrality conditions are automatically satisfied for the system under consideration. 
We find the following dispersion laws in the left handed sector,

$$
\begin{aligned}
& \epsilon_{(L) 1}=\frac{\mu_{s}}{2}-\tilde{A}_{7}+\left(2 c_{I}+c_{0}\right) \frac{\vec{v} \cdot \vec{\jmath}}{6}+\sqrt{\left(p+\frac{2 \tilde{A}_{I}}{3}-c_{7} \frac{\vec{v} \cdot \vec{\jmath}}{2}\right)^{2}+\Delta_{1}^{2}}, \\
& \epsilon_{(L) 2}=-\frac{\mu_{s}}{2}+\tilde{A}_{7}+\left(2 c_{I}+c_{0}\right) \frac{\vec{v} \cdot \vec{\jmath}}{6}+\sqrt{\left(p+\frac{2 \tilde{A}_{I}}{3}+c_{7} \frac{\vec{v} \cdot \vec{\jmath}}{2}\right)^{2}+\Delta_{1}^{2}}, \\
& \epsilon_{(L) 3}=\frac{3 \mu_{s}}{4}+\tilde{A}_{I}-\frac{\tilde{A}_{7}}{2}-A_{e}-\left(2\left(c_{I}-c_{0}\right)+3 c_{7}\right) \frac{\vec{v} \cdot \vec{\jmath}}{12} \\
& +\sqrt{\left(p+\frac{\mu_{s}}{4}-\frac{\tilde{A}_{I}}{3}-\frac{\tilde{A}_{7}}{2}+\left(2 c_{I}-c_{7}\right) \frac{\vec{v} \cdot \vec{\jmath}}{4}\right)^{2}+\Delta_{2}^{2}}, \\
& \epsilon_{(L) 4}=-\frac{3 \mu_{s}}{4}-\tilde{A}_{I}+\frac{\tilde{A}_{7}}{2}+A_{e}-\left(2\left(c_{I}-c_{0}\right)+3 c_{7}\right) \frac{\vec{v} \cdot \vec{\jmath}}{12} \\
& +\sqrt{\left(p+\frac{\mu_{s}}{4}-\frac{\tilde{A}_{I}}{3}-\frac{\tilde{A}_{7}}{2}-\left(2 c_{I}-c_{7}\right) \frac{\vec{v} \cdot \vec{\jmath}}{4}\right)^{2}+\Delta_{2}^{2}}, \\
& \epsilon_{(L) 5}=\frac{\mu_{s}}{4}+\tilde{A}_{I}+\frac{\tilde{A}_{7}}{2}-A_{e}-\left(2\left(c_{I}-c_{0}\right)-3 c_{7}\right) \frac{\vec{v} \cdot \vec{\jmath}}{12} \\
& +\sqrt{\left(p-\frac{\mu_{s}}{4}-\frac{\tilde{A}_{I}}{3}+\frac{\tilde{A}_{7}}{2}+\left(2 c_{I}+c_{7}\right) \frac{\vec{v} \cdot \vec{\jmath}}{4}\right)^{2}+\Delta_{3}^{2}}, \\
& \epsilon_{(L) 6}=-\frac{\mu_{s}}{4}-\tilde{A}_{I}-\frac{\tilde{A}_{7}}{2}+A_{e}-\left(2\left(c_{I}-c_{0}\right)-3 c_{7}\right) \frac{\vec{v} \cdot \vec{\jmath}}{12} \\
& +\sqrt{\left(p-\frac{\mu_{s}}{4}-\frac{\tilde{A}_{I}}{3}+\frac{\tilde{A}_{7}}{2}-\left(2 c_{I}+c_{7}\right) \frac{\vec{v} \cdot \vec{\jmath}}{4}\right)^{2}+\Delta_{3}^{2}}, \\
& \epsilon_{(L) 7,8,9}=\text { complicated. }
\end{aligned}
$$

The final three ("complicated") dispersion laws cannot be expressed in simple form. They are electrically neutral and do not have much impact on the dynamics, since they never become gapless for the values of $\mu_{s}$ which we will consider. The dispersion laws for the right handed sector can be obtained from the left handed ones by substituting $\tilde{A}_{7} \rightarrow-\tilde{A}_{7}$ and $c_{7} \rightarrow-c_{7}$. We note that for $c_{I}=c_{0}$ and $c_{7}=0$ the current does not shift the energy of the electrically charged modes, which is similar to the case without kaon condensate.

\section{THERMODYNAMIC POTENTIAL}

In order to evaluate the thermodynamic potential and study the phase structure we will make a number of approximations. In QCD gluons are dynamical fields, and gluon exchanges determine the value of the gap parameter. In the following we will treat the gauge fields and 


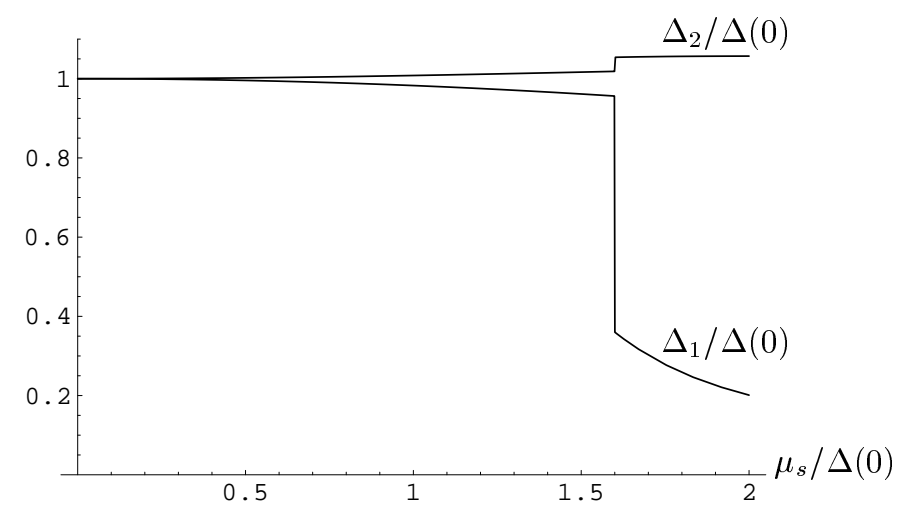

FIG. 1: Fermion gaps in the kaon condensed CFL phase as a function of the effective chemical potential $\mu_{s}$. The figure shows $\Delta_{2} / \Delta(0)$ (upper curve) and $\Delta_{1} / \Delta(0)$ (lower curve) as functions of $\mu_{s} / \Delta(0)$ for $\jmath=0$. Within our approximations $\Delta_{3}=\Delta_{2}$.

the gap as classical mean fields. The mean field partition function reproduces low energy QCD fermion propagators as well as $n$-point functions of the currents and gauge fields to leading order $O\left(\alpha_{s}^{0}\right)$ in the strong coupling constant and to $O\left(\mu_{s}^{2}\right)$ in the effective chemical potential.

In the mean field approximation the thermodynamic potential is given by

$$
\Omega=\frac{1}{G}\left(\Delta_{1}^{2}+\Delta_{2}^{2}+\Delta_{3}^{2}\right)+\frac{\left(8 c_{I}^{2}+c_{0}^{2}+6 c_{7}^{2}\right) \mu^{2} \jmath^{2}}{24 \pi^{2}}-\frac{\mu^{2}}{2 \pi^{2}} \int d p \int \frac{d \hat{v}}{4 \pi} \sum_{i=1}^{9}\left(\left|\epsilon_{i}\right|-|p|\right)-\frac{A_{e}^{4}}{12 \pi^{2}}
$$

where the first term is the mean field potential for the gap field, the second is a tadpole term that arises from integrating out anti-fermions [25], and the third term is the one-loop effective potential of fermions coupled to background gauge and gap fields. We will keep only the leading order terms in $\Delta / \mu$ in the thermodynamic potential. In particular, we have written the integral over the fermion dispersion relations in terms of the density of states, an integral $d p$ over the momentum transverse to the Fermi surface, and an angular integral $d \hat{v}$. We have included the $A_{e}^{4}$ term which is formally of higher order, but necessary to determine a unique solution to the electrical neutrality condition.

The integral over $p$ is ultraviolet divergent and requires a cutoff $\Lambda$. This divergence can be absorbed into the coupling constant $G(\Lambda)$. In the following $G$ refers to $G(\Lambda=\mu)$. The constant $G$ fixes the magnitude of the gap in the chiral limit, which we denote by $\Delta(0)$. We shall study the thermodynamic potential as a function of the dimensionless variables $\mu_{s} / \Delta(0)$ and $\jmath / \Delta(0)$. 
The integrals over $\epsilon_{1-6}$ are easily evaluated. The result is

$$
\begin{gathered}
\Omega=\frac{1}{G}\left(\Delta_{1}^{2}+\Delta_{2}^{2}+\Delta_{3}^{2}\right)+\frac{\left(8 c_{I}^{2}+c_{0}^{2}+6 c_{7}^{2}\right) \mu^{2} \jmath^{2}}{24 \pi^{2}}+R-\frac{A_{e}^{4}}{12 \pi^{2}}+ \\
+\left[F\left(\Delta_{1}, \frac{2 \tilde{A}_{I}}{3}, \frac{\mu_{s}}{2}-\tilde{A}_{7}, \frac{\jmath c_{7}}{2},\left(2 c_{I}+c_{0}\right) \frac{\jmath}{6}\right)\right. \\
+F\left(\Delta_{2}, \frac{\mu_{s}}{4}+\frac{\tilde{A}_{I}}{3}+\frac{\tilde{A}_{7}}{2}, \frac{3 \mu_{s}}{4}+\tilde{A}_{I}-\frac{\tilde{A}_{7}}{2}-A_{e},\left(2 c_{I}-c_{7}\right) \frac{\jmath}{4}\right. \\
\left.\quad\left(2\left(c_{I}-c_{0}\right)+3 c_{7}\right) \frac{\jmath}{12}\right) \\
+F\left(\Delta_{3}, \frac{\mu_{s}}{4}-\frac{\tilde{A}_{I}}{3}+\frac{\tilde{A}_{7}}{2}, \frac{\mu_{s}}{4}+\tilde{A}_{I}+\frac{\tilde{A}_{7}}{2}-A_{e},\left(2 c_{I}+c_{7}\right) \frac{\jmath}{4}\right. \\
\left.\left.\left(2\left(c_{I}-c_{0}\right)-3 c_{7}\right) \frac{\jmath}{12}\right)+\left(\tilde{A}_{7} \leftrightarrow-\tilde{A}_{7}, c_{7} \leftrightarrow-c_{7}\right)\right]
\end{gathered}
$$

where $R$ is the integral of the complicated modes,

$$
R=-\frac{\mu^{2}}{2 \pi^{2}} \int d p \int \frac{d \hat{v}}{4 \pi} \sum_{i=7}^{9}\left(\left|\epsilon_{i}\right|-|p|\right) .
$$

The function $F\left(\Delta, a_{1}, a_{2}, \jmath_{1}, \jmath_{2}\right)$ is defined by

$$
\begin{gathered}
F\left(\Delta, a_{1}, a_{2}, \jmath_{1}, \jmath_{2}\right)=-\frac{\mu^{2}}{12 \pi^{2}}\left[3 \Delta^{2}\left(1+2 \log \left(\frac{2 \mu}{\Delta}\right)\right)+6 a_{1}^{2}+2 \jmath_{1}^{2}\right] \\
+\frac{\mu^{2}}{24 \pi^{2}}\left\{\Theta ( a _ { 2 } - \jmath _ { 2 } - \Delta ) \frac { 1 } { \jmath _ { 2 } } \left[\lambda\left(\jmath_{2}\right)\left(\lambda\left(\jmath_{2}\right)^{2}+3 \Delta^{2}\right)\right.\right. \\
\left.\left.+3 \Delta^{2}\left(a_{2}-\jmath_{2}\right) \log \left(\frac{a_{2}-\jmath_{2}-\lambda\left(\jmath_{2}\right)}{a_{2}-\jmath_{2}+\lambda\left(\jmath_{2}\right)}\right)\right]+\left(\jmath_{2} \leftrightarrow-\jmath_{2}\right)\right\},
\end{gathered}
$$

with $\lambda\left(\jmath_{2}\right)=\sqrt{\left(a_{2}-\jmath_{2}\right)^{2}-\Delta^{2}}$. Here, $a_{1}$ and $\jmath_{1}$ refer to the shift in the minimum of the dispersion relation due to the gauge potentials and the current, and $a_{2}$ and $\jmath_{2}$ denote the shift in the energy. The equations for color and electrical neutrality and the equation $\partial \Omega / \partial \Delta_{2}=\partial \Omega / \partial \Delta_{3}=0$ are solved by

$$
\begin{gathered}
\tilde{A}_{I}=\tilde{A}_{7}=0, \quad \Delta_{3}=\Delta_{2}, \\
A_{e}=\max \left(0,-\Delta_{2}+\frac{3 \mu_{s}}{4}+\left(2\left|c_{I}-c_{0}\right|+3\left|c_{7}\right|\right) \frac{\jmath}{12}\right),
\end{gathered}
$$

which greatly simplifies the expression for the thermodynamic potential. The remaining equations for $\Delta_{1}, \Delta_{2}$ and $\jmath$ are solved by numerical minimization of the thermodynamic potential.

First let us consider the case $\jmath=0$. Fig. 1 shows the solution of the gap equations. We notice that even for small $\mu_{s}$ there is a small splitting between $\Delta_{1}$ and $\Delta_{2,3}$. The 


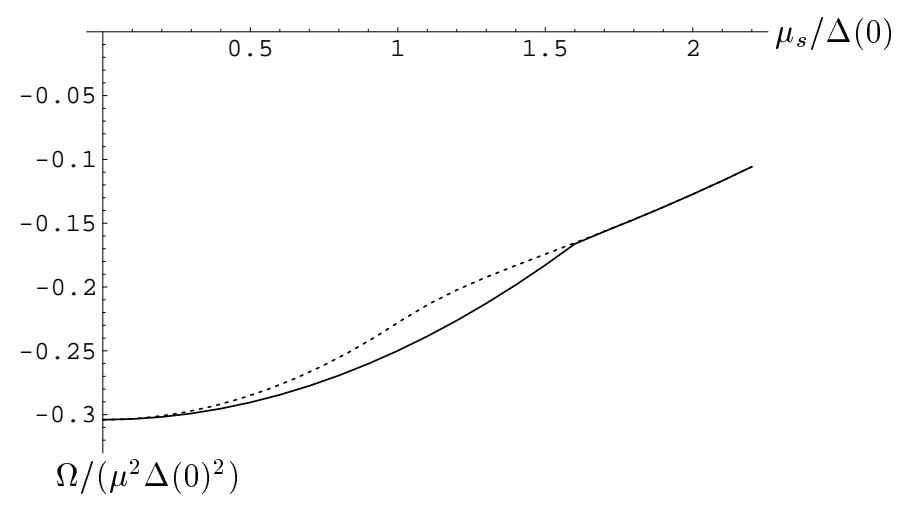

FIG. 2: Thermodynamic potential of the kaon condensed CFL phase $\Omega /\left(\mu^{2} \Delta(0)^{2}\right)$ relative to the thermodynamic potential of neutral non-interacting quark matter as a function of $\mu_{s} / \Delta(0)$ for $\jmath=0$. For comparison we also show the thermodynamic potential of the gCFL phase using the same approximations (dotted line).

thermodynamic potential is shown in Fig. 2, where we also compare to the result for the CFL-gCFL phase without a kaon condensate. We find that $\epsilon_{4}$ becomes quadratic in $p$ for $\mu_{s}>\mu_{s}^{(1)}=1.35 \Delta(0)$, and that the mode $\epsilon_{2}$ becomes gapless for $\mu_{s}>\mu_{s}^{(2)}=1.607 \Delta(0)$. In contrast to the CFL-gCFL phase transition, the phase transition at $\mu_{s}^{(2)}$ is first order. At $\mu_{s}=\mu_{s}^{(2)}$ there is a substantial drop in $\Delta_{1}$ and a small increase in $\Delta_{2,3}$. The thermodynamic potential is smooth at $\mu_{s}^{(1)}$ but has a kink at $\mu_{s}^{(2)}$.

We observe that near $\mu_{s}^{(2)}$ the thermodynamic potential of the gCFLK phase is very close to the thermodynamic potential of the gCFL phase $\left(\Omega_{g C F L K}<\Omega_{g C F L}\right.$ by a very small amount). It is not entirely clear why this is the case. Near $\mu_{s}^{(2)}$ the gauge potentials become quite large and our approximations are breaking down. Also, since some of the gaps become quite small it is not clear whether the correct CFLK state is a simple flavor rotation of the CFL state. We note that near $\mu_{s}^{(2)}$ the thermodynamic potential in the gCFL phase plotted in Fig. 1 is lower than the result of Alford et al. [7]. This difference is also due to higher order terms. In the gCFL phase the thermodynamic potential in equ. (13) differs from the functional used by Alford et al. by terms of $O\left(\mu_{s}^{4}\right)$.

Next we study the effect of a Goldstone boson current. We first consider the region $\mu_{s} \simeq$ $\mu_{s}^{(1)}$, which was also investigated in [13, 14]. If we take into account the condition of electrical neutrality we find that the expectation value of the current becomes extremely small. The competition between the $A_{e}^{4}$ and $\mu^{2} \jmath^{2}$ terms leads to a minimum of $\Omega$ at $\jmath \sim\left(\frac{3}{4} \mu_{s}-\Delta_{2}\right)^{3} / \mu^{2}$, 


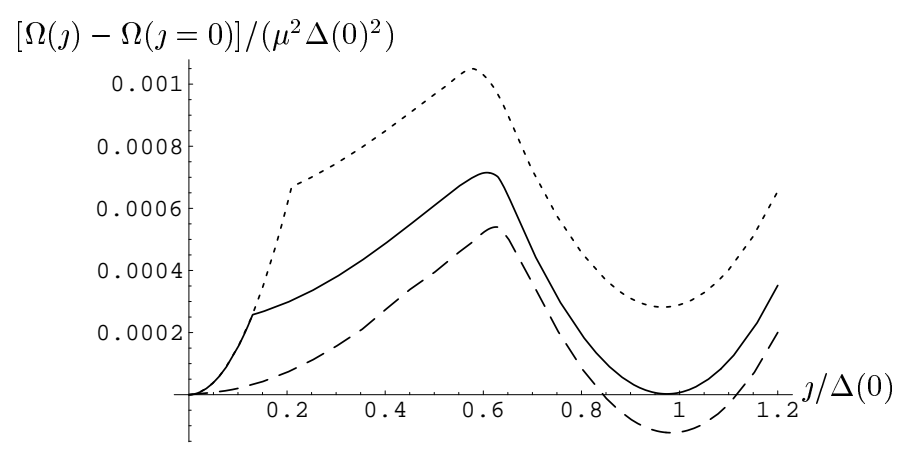

FIG. 3: Thermodynamic potential as a function of the Goldstone boson current near $\mu_{s}^{(2)}$. The figure shows $[\Omega(\jmath)-\Omega(\jmath=0)] /\left(\mu^{2} \Delta(0)^{2}\right)$ at $\mu_{s}=1.600 \Delta(0)$ (dotted), $\mu_{s}=1.605 \Delta(0)$ (continuous) and $\mu_{s}=1.610 \Delta(0)$ (dashed).

corresponding to a contribution to $\Omega$ of the order $\left(\frac{3}{4} \mu_{s}-\Delta_{2}\right)^{6} / \mu^{2}$. A detailed calculation of the current is presented in the appendix. Since the current is so small, we shall neglect its effect in the remainder of this section.

Now consider the region $\mu_{s} \simeq \mu_{s}^{(2)}$. Here the optimal value for $c_{7}$ turns out to be zero, because $c_{7}$ does not contribute to the shift of the energy of $\epsilon_{2}$. If we set $c_{I}=c_{0}$ the current shifts only the energy of the electrically neutral mode. This should be the preferred current if electrical neutrality is enforced. We set $c_{I}=1.2$ and $c_{0}=1$, which leads to an even stronger minimum of the thermodynamic potential at finite $\jmath$.

Fig. 3 shows the thermodynamic potential as a function of $\jmath$ for different values of $\mu_{s}$ in the vicinity of $\mu_{s}=1.6 \Delta(0)$. The kinks come from a discontinuity in $\Delta_{1,2}(\mathrm{\jmath})$, similar to the discontinuity in $\Delta_{1,2}\left(\mu_{s}\right)$ shown in Fig. 1. We observe that the thermodynamic potential develops a nontrivial minimum if $\mu_{s}>1.605 \Delta(0)$, which is slightly below the value of $\mu_{s}$ where the mode $\epsilon_{2}$ becomes gapless at zero current. We remark that the window where the thermodynamic potential has a nontrivial minimum is rather small. For $\mu_{s}$ slightly above $1.615 \Delta(0)$ we find that the global minimum is again at $\jmath=0$. We note, however, that the some of the gaps are very small, the current at the non-trivial minimum is rather large, and our approximations are not reliable. Since the currents are large the correct ground state may well be of the LOFF type and involve multiple currents and complicated crystal structures as in [26]. In addition to that, inhomogeneities of the amplitude of the order parameter may play a role [27, 28]. 


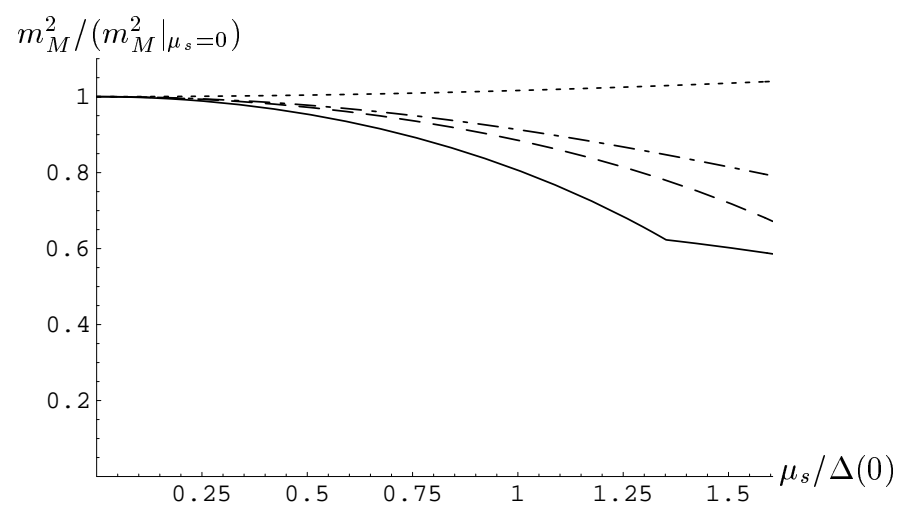

FIG. 4: Magnetic gluon screening (Meissner) masses in the kaon condensed CFL phase as a function of the effective chemical potential $\mu_{s}$. The figure shows squared Meissner masses $m_{M 11}^{2}=m_{M 22}^{2}=$ $m_{M 44}^{2}=m_{M 55}^{2}$ (continuous), $m_{M 66}^{2}=m_{M(1)}^{2}$ (dashed), $m_{M 77}^{2}$ (dotted), $m_{M(2)}^{2}$ (dash-dotted) in units of $\left.m_{M}^{2}\right|_{\mu_{s}=0} \equiv(21-8 \log 2) g^{2} \mu^{2} /\left(108 \pi^{2}\right)$ as functions of $\mu_{s} / \Delta(0)$.

\section{MEISSNER MASSES}

In this section we shall study the stability of the CFLK and gCFLK phase with and without a Goldstone boson current with regard to chromomagnetic instabilities. At leading order in $\alpha_{s}$ the screening masses are given by a one-loop diagram involving Nambu-Gor'kov propagators. We have

$$
\begin{aligned}
\left(m_{M}^{2}\right)_{a b}^{i j}= & \frac{g^{2} \mu^{2}}{2 \pi^{2}} \delta_{a b} \delta^{i j}+\frac{g^{2} \mu^{2}}{16 \pi^{2}} \lim _{\vec{k} \rightarrow 0} \lim _{k_{0} \rightarrow 0} \int d p \int \frac{d \hat{v}}{4 \pi} \int \frac{d p_{0}}{2 \pi} \\
& \times \operatorname{Tr}\left[G^{+}(p) V_{(L) a}^{i} G^{+}(p+k) V_{(L) b}^{j}+G^{-}(p) \tilde{V}_{(L) a}^{i} G^{-}(p+k) \tilde{V}_{(L) b}^{j}\right. \\
& \left.+\Xi^{+}(p) V_{(L) a}^{i} \Xi^{-}(p+k) \tilde{V}_{(L) b}^{j}+\Xi^{-}(p) \tilde{V}_{(L) a}^{i} \Xi^{+}(p+k) V_{(L) b}^{j}+(R \leftrightarrow L)\right]
\end{aligned}
$$

where we have defined the vertices

$$
\begin{array}{ll}
\left(V_{(L) a}^{i}\right)_{A B}=\frac{1}{2} \operatorname{Tr}\left[\lambda_{A} \lambda_{B} X \lambda_{a}^{T} X^{\dagger}\right] v^{i}, & \left(\tilde{V}_{(L) a}^{i}\right)_{A B}=-\frac{1}{2} \operatorname{Tr}\left[\lambda_{B} \lambda_{A} X \lambda_{a}^{T} X^{\dagger}\right] \tilde{v}^{i}, \\
\left(V_{(R) a}^{i}\right)_{A B}=\frac{1}{2} \operatorname{Tr}\left[\lambda_{A} \lambda_{B} X^{\dagger} \lambda_{a}^{T} X\right] v^{i}, & \left(\tilde{V}_{(R) a}^{i}\right)_{A B}=-\frac{1}{2} \operatorname{Tr}\left[\lambda_{B} \lambda_{A} X^{\dagger} \lambda_{a}^{T} X\right] \tilde{v}^{i},
\end{array}
$$

with $\tilde{v}^{i}=-v^{i}$. We remark that right handed and left handed quark propagators are identical because of $\tilde{A}_{7}=c_{7}=0$.

We find that the mass matrix in the 3-8-sector is not diagonal, the mixing angle being equal to $\pi / 6$. We denote the corresponding eigenvalues by $m_{M(1)}^{2}$ and $m_{M(2)}^{2}$. We find that 


\begin{tabular}{|c||c|c|c|c|}
\hline & $m_{M 11}^{2}$ & $m_{M 66}^{2}$ & $m_{M 77}^{2}$ & $m_{M(2)}^{2}$ \\
\hline \hline transv. & 0.16 & 0.59 & 1.09 & 0.21 \\
\hline long. & 0.31 & 0.87 & 1.05 & 0.46 \\
\hline
\end{tabular}

TABLE I: Meissner masses at the minimum of $\Omega(\jmath)$ for $\mu_{s}=1.605 \Delta(0)$, in units of $\left.m_{M}^{2}\right|_{\mu_{s}=0}$.

some of the Meissner masses are equal,

$$
m_{M 11}^{2}=m_{M 22}^{2}=m_{M 44}^{2}=m_{M 55}^{2}, \quad m_{M 66}^{2}=m_{M(1)}^{2}
$$

Fig. 4 shows the Meissner masses as functions of $\mu_{s}$ for $\mu_{s}<\mu_{s}^{(2)}=1.607 \Delta(0)$. The kink in $m_{M 11}$ at $\mu_{s}=\mu_{s}^{(1)}$ results from the kink in $A_{e}$. We observe that the Meissner masses in the gapless CFLK phase are real, even in the absence of a current.

For $\mu_{s}>\mu_{s}^{(2)}$ and zero current the Meissner masses $m_{M 11}^{2}=m_{M 22}^{2}=m_{M 44}^{2}=m_{M 55}^{2}$ as well as $m_{M(1)}^{2}$ receive a negative contributions proportional to $-1 / \sqrt{\mu_{s}^{2}-4 \Delta_{1}^{2}}$. This is the usual chromomagnetic instability for zero current. In the presence of a finite current $\vec{\jmath}$ we may decompose the Meissner masses into a longitudinal and a transverse component,

$$
\left(m_{M}^{2}\right)^{i j}=m_{M \perp}^{2}\left(\delta^{i j}-\hat{\jmath}^{i} \hat{\jmath}^{j}\right)+m_{M \|}^{2} \hat{\jmath}^{i} \hat{\jmath}^{j}
$$

The values of the Meissner masses at finite current just above the phase transition at $\mu_{s}=$ $1.605 \Delta(0)$ are shown in Table I. We note that all these values are positive. We note that for $\mu_{s}>1.615 \Delta(0)$ there is no Goldstone boson current and the magnetic screening masses in the CFLK phase are tachyonic. This is an unusual situation because stability with respect to a Goldstone boson current implies that the phase is stable with respect to electrically neutral fluctuations of the gauge field. We will comment on this regime in the conclusions.

\section{CONCLUSIONS}

We have studied the stability of a kaon condensed CFL phase with respect to the formation of a Goldstone boson current. We have computed the thermodynamic potential and the gluon screening masses as a function of the effective chemical potential $\mu_{s}=m_{s}^{2} /\left(2 p_{F}\right)$. Our starting point is an effective lagrangian of fermions coupled to a mean-field gap term and background gauge potentials. Our methods are reliable provided the gauge potentials and currents are smaller than the gaps. There are three important scales that appear in 
the problem, $\mu_{s}=m_{K}$ is the onset of kaon condensation, $\mu_{s}=\mu_{s}^{(1)}=1.35 \Delta(0)$ is the point where a charged fermion mode becomes (almost) gapless, and $\mu_{s}=\mu_{s}^{(2)}=1.61 \Delta(0)$ is the point where a neutral fermion mode becomes gapless. The CFLK-gCFLK transition at $\mu_{s}^{(1)}$ is smooth while the transition at $\mu_{s}^{(2)}$ is first order.

We find that the kaon condensed CFL phase is more stable with respect to chromomagnetic instabilities than the CFL phase [29, 30]. The gluon screening masses are real in the regime $\mu_{s}<\mu_{s}^{(2)}$, despite the presence of an almost gapless mode. There is a weak Goldstone boson current instability for $\mu_{s}>\mu_{s}^{(1)}$. The magnitude of the current is suppressed by the constraint of electric charge neutrality.

We find that the gluon screening masses become imaginary in the CFLK phase for $\mu_{s}>$ $\mu_{s}^{(2)}$. There is a Goldstone boson current instability that develops at this point, and the screening masses in the presence of a Goldstone boson current are positive. However, the Goldstone boson current phase only exists in a very small window above $\mu_{s}^{(2)}$. Beyond that point the CFLK phase is stable with respect to the formation of Goldstone boson currents, but the gluon screening masses are imaginary. This is somewhat puzzling since the Goldstone boson currents are equivalent to non-zero gauge fields, see equ. (9). The difference between the second derivative of the thermodynamic potential with respect to the Goldstone boson currents and the screening masses is that the former are computed at constant (zero) electric charge, while the latter are computed at constant electro-static potential. In this sense stability with respect to Goldstone boson currents is the physically relevant criterion. The fact that the Meissner masses are imaginary may nevertheless imply that other instabilities are present. In this regime some of the gaps are small compared the background fields and it may be necessary to consider states with multiple currents, such as the crystalline LOFF state [18, 20, 26].

Acknowledgments: This work is supported in part by the US Department of Energy grant DE-FG-88ER40388 (T.S. and A.G.) and DE-FG02-87ER40365 as well as the National Science Foundation grant PHY-0244822 (A.K.). We would like to thank J. Kapusta for pointing out a sign mistake in the second part of equ. (23) in [21]. 


\section{APPENDIX A: CHARGE NEUTRALITY AND GOLDSTONE BOSON CUR-} RENT FOR $\mu_{s}>\mu_{s}^{(1)}$

In this appendix we study in more detail the current instability in the regime $\mu_{s}>\mu_{s}^{(1)} \simeq$ $4 \Delta / 3$. For simplicity we only consider a pure hypercharge current with $V=1$. Including the baryon current will only change some numerical coefficients. Our analysis extends the

analysis of [13, 14] to properly implement charge neutrality in the Goldstone boson current state.

The instability is driven by the lowest fermion mode in the spectrum. The dispersion relation is given by

$$
\omega_{p}=\Delta+\frac{\left(p-l_{0}\right)^{2}}{2 \Delta}-\frac{3}{4} \mu_{s}+A_{e}-\frac{1}{4} \vec{v} \cdot \vec{\jmath}_{K}
$$

where $A_{e}$ is the electron chemical potential, $\mu_{s}=m_{s}^{2} /\left(2 p_{F}\right)$ and $l_{0}=\left(\mu_{s}-\vec{v} \cdot \vec{\jmath}_{K}\right) / 4$. The contribution of gapless modes to the thermodynamic potential is

$$
\Omega=-2 \frac{\mu^{2}}{2 \pi^{2}} \int d p \int \frac{d \hat{v}}{4 \pi} \omega_{p} \theta\left(-\omega_{p}\right),
$$

where the factor 2 is a degeneracy factor and $d \hat{v}$ is an integral over the Fermi surface. The charge density of gapless modes is

$$
q=2 \frac{\mu^{2}}{2 \pi^{2}} \int d p \int \frac{d \hat{v}}{4 \pi} \theta\left(-\omega_{p}\right)
$$

The electron contribution to the thermodynamic potential and the charge density is

$$
\Omega_{e}=-\frac{A_{e}^{4}}{12 \pi^{2}}, \quad q_{e}=-\frac{A_{e}^{3}}{3 \pi^{2}}
$$

We introduce dimensionless variables

$$
x=\frac{\jmath_{K}}{a \Delta}, \quad h=\frac{3 \mu_{s}-4 \Delta}{a \Delta}, \quad h_{e}=\frac{4 A_{e}}{a \Delta},
$$

where $a$ is a numerical coefficient that depends on the parameters of the effective theory and the number of currents that are turned on. For a pure kaon current

$$
a=\frac{2}{15^{2} c_{\pi}^{2} v_{\pi}^{4}},
$$

where $c_{\pi}=(21-8 \log (2)) / 36$ is the numerical constant that appears in the weak coupling result for the pion decay constant $f_{\pi}$ and $v_{\pi}^{2}=1 / 3$ is the square of the Goldstone boson 
velocity. The thermodynamic potential and charge density can be written as

$$
\begin{aligned}
\Omega\left(h, h_{e}, x\right) & =\frac{\mu^{2} \Delta^{2}}{\pi^{2}}\left(C f_{h-h_{e}}(x)-C_{e} h_{e}^{4}\right), \\
q\left(h, h_{e}, x\right) & =\frac{\mu^{2} \Delta}{\pi^{2}}\left(K g_{h-h_{e}}(x)-K_{e} h_{e}^{3}\right)
\end{aligned}
$$

with

$$
\begin{aligned}
& f_{h}(x)=x^{2}-\frac{1}{x}\left[(h+x)^{5 / 2} \Theta(h+x)-(h-x)^{5 / 2} \Theta(h-x)\right], \\
& g_{h}(x)=\frac{1}{x}\left[(h+x)^{3 / 2} \Theta(h+x)-(h-x)^{3 / 2} \Theta(h-x)\right] .
\end{aligned}
$$

The numerical coefficients are given by

$$
C=\frac{2}{15^{4} c_{\pi}^{3} v_{\pi}^{6}} \frac{\mu^{2} \Delta^{2}}{\pi^{2}}, \quad K=\frac{10 C}{a}
$$

and

$$
C_{e}=\frac{1}{3 \cdot 4^{5}}\left(\frac{\Delta^{2}}{\mu^{2}}\right), \quad K_{e}=\frac{16 C_{e}}{a} .
$$

We first solve the neutrality condition in order to find $h_{e}=h_{e}(h, x)$. We have $h_{e}=0$ if $h+x<0$. For $h+x>0$ we can use $K_{e} \ll 1$ in order to write

$$
h_{e}=h+x+\delta h_{e}
$$

with $\delta h_{e} \ll h_{e}$. We get

$$
\delta h_{e}=\left(\frac{K_{e}}{K}\right)^{2 / 3} x^{2 / 3}(h+x)^{2} .
$$

Inserting this result into the energy density gives

$$
\Omega=\frac{\mu^{2} \Delta^{2}}{\pi^{2}}\left\{C\left[x^{2}-\frac{1}{x} \delta h_{e}^{5 / 2} \Theta\left(\delta h_{e}\right)\right]-C_{e}(h+x)^{4}+\ldots\right\} .
$$

The contribution from gapless fermions is proportional to $x^{2 / 3}$ for small $x$ so the instability is still present. If $K_{e}, C_{e} \ll 1$ the minimum of the thermodynamic potential is determined by the balance between the electron term and the current contribution. We get

$$
x=\frac{2 C_{e}}{C} h^{3} \sim\left(\frac{\Delta^{2}}{\mu^{2}}\right) h^{3} .
$$

This implies that a Goldstone current is formed, but the magnitude of the current is suppressed as compared to the result given in [13, 14].

[1] M. Alford and K. Rajagopal, hep-ph/0606157; M. Huang, hep-ph/0602092; S. B. Ruster, V. Werth, M. Buballa, I. A. Shovkovy and D. H. Rischke, nucl-th/0602018; T. Schäfer, 
nucl-th/0602067; in "Pairing in fermionic systems: Basic concepts and modern applications", Series on Advances in Quantum Many-Body Theory, Vol. 8, World Scientific Publishing, Singapore (2007).

[2] M. Alford, K. Rajagopal and F. Wilczek, Nucl. Phys. B537, 443 (1999) hep-ph/9804403.

[3] T. Schäfer, Nucl. Phys. B 575, 269 (2000) hep-ph/9909574.

[4] N. Evans, J. Hormuzdiar, S. D. Hsu and M. Schwetz, Nucl. Phys. B 581, 391 (2000) hep-ph/9910313.

[5] P. F. Bedaque and T. Schäfer, Nucl. Phys. A 697, 802 (2002) hep-ph/0105150.

[6] M. Alford, C. Kouvaris and K. Rajagopal, Phys. Rev. Lett. 92, 222001 (2004) hep-ph/0311286.

[7] M. Alford, C. Kouvaris and K. Rajagopal, Phys. Rev. D 71, 054009 (2005) hep-ph/0406137.

[8] S.-T. Wu, S. Yip, Phys. Rev. A 67, 053603 (2003) cond-mat/0303185].

[9] M. Huang and I. A. Shovkovy, Phys. Rev. D 70, 051501 (2004) hep-ph/0407049].

[10] R. Casalbuoni, R. Gatto, M. Mannarelli, G. Nardulli and M. Ruggieri, Phys. Lett. B 605, 362 (2005) [Erratum-ibid. B 615, 297 (2005)] hep-ph/0410401].

[11] M. Alford and Q. H. Wang, J. Phys. G 31, 719 (2005) hep-ph/0501078.

[12] K. Fukushima, Phys. Rev. D 72, 074002 (2005) hep-ph/0506080].

[13] A. Kryjevski, preprint, hep-ph/0508180.

[14] T. Schäfer, Phys. Rev. Lett. 96, 012305 (2006) hep-ph/0508190.

[15] A. Gerhold and T. Schäfer, Phys. Rev. D 73, 125022 (2006) hep-ph/0603257.

[16] M. G. Alford, J. A. Bowers and K. Rajagopal, Phys. Rev. D 63, 074016 (2001) hep-ph/0008208.

[17] R. Casalbuoni and G. Nardulli, Rev. Mod. Phys. 76, 263 (2004) hep-ph/0305069.

[18] R. Casalbuoni, R. Gatto, N. Ippolito, G. Nardulli and M. Ruggieri, Phys. Lett. B 627, 89 (2005) hep-ph/0507247.

[19] M. Ciminale, G. Nardulli, M. Ruggieri and R. Gatto, Phys. Lett. B 636, 317 (2006) hep-ph/0602180.

[20] M. Mannarelli, K. Rajagopal and R. Sharma, Phys. Rev. D 73, 114012 (2006) hep-ph/0603076.

[21] A. Kryjevski and T. Schäfer, Phys. Lett. B 606, 52 (2005) hep-ph/0407329.

[22] A. Kryjevski and D. Yamada, Phys. Rev. D 71, 014011 (2005) hep-ph/0407350. 
[23] R. Casalbuoni and R. Gatto, Phys. Lett. B 464, 111 (1999) [hep-ph/9908227].

[24] A. Kryjevski, Phys. Rev. D 68, 074008 (2003) hep-ph/0305173.

[25] D. T. Son and M. Stephanov, Phys. Rev. D61, 074012 (2000) hep-ph/9910491,

[26] K. Rajagopal and R. Sharma, Phys. Rev. D 74, 094019 (2006) hep-ph/0605316.

[27] K. Iida and K. Fukushima, Phys. Rev. D 74, 074020 (2006) hep-ph/0603179.

[28] I. Giannakis, D. Hou, M. Huang and H. c. Ren, preprint, hep-ph/0606178.

[29] X. B. Zhang and J. I. Kapusta, preprint, hep-ph/0605138; preprint, hep-ph/0612135.

[30] H. J. Warringa, preprint, hep-ph/0606063. 